\title{
Dominance of General versus Specific Aspects of Wellbeing on the Student Subjective Wellbeing
}

\section{Questionnaire}

\author{
Anthony J. Roberson ${ }^{1}$ and Tyler L. Renshaw ${ }^{2}$ \\ ${ }^{1}$ Department of Clinical, Health, and Applied Sciences, University of Houston-Clear Lake \\ ${ }^{2}$ Department of Psychology, Utah State University
}

\begin{abstract}
Author Note
Data collection was sponsored and facilitated by the Character Lab Research Network (CLRN; https://characterlab.org/research-network/). We are grateful for the generosity of CLRN staff, their partner schools, and participating students in making this project possible.

Supporting materials including data and $\mathrm{R}$ analysis scripts are freely available on the Open Science Framework (https://osf.io/exyb7/?view_only=91aea92910d444999f95555beaba5846).

The authors have no conflicts of interest to disclose.

Correspondence concerning this article should be addressed to Anthony J. Roberson, University of Houston-Clear Lake, Department of Clinical, Health, and Applied Sciences, 2700 Bay Area Boulevard, Houston, TX 77058. Email: robersona@uhcl.edu
\end{abstract}

(c) 2022, American Psychological Association. This paper is not the copy of record and may not exactly replicate the final, authoritative version of the article. Please do not copy or cite without authors' permission. The final article will be available, upon publication, via its DOI: $10.1037 / \mathrm{spq} 0000513$ 


\section{Abstract}

The Student Subjective Wellbeing Questionnaire (SSWQ) is a 16-item measure of school-specific subjective wellbeing intended for mental health screening applications. We extended past validation work to scrutinize the SSWQ's previously proposed factor structure (i.e., four group factors; one general factor) and score reliability using two random subsets of data from a sample of 1,020 adolescents in grades 9-12. Results from exploratory and confirmatory factor analyses suggested that the four items related to the educational purpose [EP] group factor did not cohere as expected, unlike the items for the other three group factors. Revised 12-item models that excluded the EP items were defensible across both subsamples. Model-based reliability indices for the 12 -item general factor scores were consistently strong and showed exceptionally high correlation with the original 16 -item version $(r=.98)$, yet unique reliability of the group factor scores was comparatively weaker. We recommend future research reevaluate the SSWQ item content and factor structure and consider using model-based factor scores at least in addition to sum scores for analyses when operating in a latent factor framework. We emphasize relying on the general rather than the group factor scores to parse individual differences in student subjective wellbeing or to make critical decisions regarding intervention resource allocation.

Keywords: subjective wellbeing, screening, school mental health, measurement, assessment

\section{IMPACT STATEMENT}

We found that youth-reported subjective wellbeing specifically related to school could be measured with fewer questions than it has previously. Although this measure gives information about different kinds of school wellbeing, we recommend that professionals stick to only looking at the general total score if making important choices about student mental health as it is the strongest indicator overall and simplifies decision making. 
DOMINANCE OF GENERAL VERSUS SPECIFIC ASPECTS OF WELLBEING ON THE STUDENT SUBJECTIVE

\section{WELLBEING QUESTIONNAIRE}

Screening for students' subjective wellbeing is an emerging practice for informing data-based decision making within school mental health service delivery frameworks. Subjective wellbeing refers to self-perceptions of how well one is functioning in life, especially in areas that are personally, socially, or culturally desirable (Renshaw et al., 2015). In the early days of researching this construct, subjective wellbeing was considered synonymous with happiness and was operationalized through measurement models targeting life satisfaction and positive affect (Diener, 1984). Measurement models have evolved and broadened over time, now accounting for a variety of desirable experiential domains, including positive emotion, activity engagement, fulfilling relationships, meaning or purpose, and accomplishment (e.g., Seligman's [2018] PERMA model). Some measurement models have also become increasingly particular, moving from general indicators of subjective wellbeing (e.g., Diener's [1985] Satisfaction with Life Scale) to multidimensional (e.g., Huebner's [1994] Multidimensional Students' Life Satisfaction Scale [MSLSS]) and context- and domain-specific indicators (e.g., Appleton's [2006] Student Engagement Instrument).

Models of Subjective Wellbeing in the School Context

As subjective wellbeing models have become more domain-specific, there has been growing interest in assessing and promoting youth subjective wellbeing in schools. Evidence from interdisciplinary researchers suggests that youth subjective wellbeing has meaningful associations with academic success (Bücker et al., 2018) and prosocial behaviors at school (Tian et al., 2015), and that school-specific subjective wellbeing is valuable as an outcome for its own sake (Bird \& Markle, 2012). Moreover, reviews of community- and school-based intervention research show that various psychosocial programs are capable of producing small to moderate improvements in students' subjective wellbeing (e.g., Carr et al., 2021; Shankland \& Rosset, 2017). 
There are at least four intersecting disciplines that have contributed to our current

understanding of youth subjective wellbeing in schools. The first and most foundational discipline is positive psychology (PP), which has been described as the science of positive states, traits, and institutions (Seligman \& Csikszentmihalyi, 2000). PP is generally credited with establishing empirical approaches to measuring and intervening with subjective wellbeing, which have since been adopted and expanded by other disciplines. PP also gave rise to the so-called "positive education" movement, which has become an international model for promoting wellbeing in schools (Seligman et al., 2009; Seligman \& Adler, 2018). The second relevant discipline is positive youth development (PYD), which integrates positive psychology within an applied developmental framework that focuses on cultivating strengths and assets through intervening with key developmental systems (Lerner et al., 2005). Within this field, subjective wellbeing is targeted as a critical internal strength or asset and schools are targeted as key systems for promoting such strengths and assets. Another related discipline is social-emotional learning (SEL), which aims to train general social and emotional competencies (e.g., self-awareness, responsible decision making) in schools for the purposes of improving broad student outcomes, including subjective wellbeing (Weissberg et al., 2015).

The final intersecting discipline is school mental health $(\mathrm{SMH})$, which is a population-based, multitiered, collaborative approach to preventing mental health problems and promoting wellbeing in schools (Weist et al., 2013). Our framing of youth subjective wellbeing for this study is primarily informed by the SMH discipline, which does not propose a unique approach to youth wellbeing per se, but rather centers on applying what the other three disciplines (i.e., PP, PYD, SEL) share in common: the proposition that youth subjective wellbeing is a valuable outcome, which can be conceptualized, measured, and promoted in schools.

More particularly, this study is grounded in a core concept emerging within the SMH tradition known as complete mental health. Proponents of this model posit that mental health can be usefully thought of as a bidimensional construct that includes an undesirable dimension-defined by 
psychopathology, distress, or problem behavior-and a desirable dimension-defined by wellbeing, prosociality, or other healthy behavior (Suldo et al., 2016).

\section{The Complete Mental Health Paradigm}

Within $\mathrm{SMH}$, the complete mental health concept has typically been operationalized through measurement models intended to guide population-based screening (e.g., Moore et al., 2015). Findings from a growing number of studies in this area suggest that, compared to traditional screening models (i.e., problem-, distress-, psychopathology-only), classification schemes measuring both problem and wellbeing constructs add value in (a) identifying students with differing risk profiles, (b) triaging students by overall risk level, (c) predicting school functioning and adjustment, and (d) matching appropriate targeted (or Tier 2) supports post-screening (e.g., Moore, Dowdy et al., 2019; Moore, Mayworm et al., 2019). Considering the evidence, the complete mental health concept appears to have practical promise for bolstering mental health promotion in schools. Yet there remain scholarly barriers preventing more widespread adoption of such models.

One particularly salient barrier is the lack of consensus regarding how to best operationalize the wellbeing dimension in complete mental health measurement. There is longstanding agreement within the youth and school mental health fields regarding how to operationalize problem behavior or psychopathology: measuring the general domains of internalizing and externalizing problems or their more specific subdomains, such as anxiety, depression, conduct problems, oppositional defiance, and hyperactivity-impulsivity (Whitcomb, 2018). Yet even a cursory review of the literature shows far less agreement when it comes to wellbeing.

For example, complete mental health researchers have previously assessed the wellbeing dimension using single general indicators (e.g., ratings of overall life satisfaction; Renshaw \& Cohen, 2014), combinations of two or more general indicators (e.g., ratings of life satisfaction and positive affect; Suldo \& Shaffer, 2008), multidimensional measures of several general and specific indicators

(e.g., gratitude, zest, optimism, persistence, and covitality gauged by the Social Emotional Health Survey; 
Moore, Dowdy et al., 2019), and informant-reported multidimensional measures of key domain-specific

indicators (e.g., desirable social, emotional, academic, and overall behavior in the classroom as measured on the Student Wellbeing Teacher Report Scale; Roberson \& Renshaw, 2019). These operationalizations have been derived from the different disciplines contributing to youth subjective wellbeing mentioned above (i.e., PP, PYD, SEL) and have largely been based on researchers' preferred models rather than empirical considerations, as there is not yet a comprehensive evidence base supporting preference for one discipline or measurement approach over another. Moreover, from a pragmatic perspective, determining whether a domain-general or a domain-specific approach is globally superior to the other in understanding subjective wellbeing, regardless of intended use, is not the goal. Instead, we contend these approaches are complementary, each covering distinct views of youth wellbeing, and therefore could be more or less useful depending on the intended purpose of their adoption (see Zhang \& Renshaw, 2020).

We contribute to this discussion by further investigating the defensibility of operationalizing student wellbeing via self-reports of school-specific positive psychological functioning. As noted above, our framing of this study is not grounded squarely in PP, PYD, or SEL disciplines; rather, it is grounded in the $\mathrm{SMH}$ approach to recognizing the common ground between them: the proposition that youth subjective wellbeing is a valuable outcome, which can be conceptualized, measured, and promoted in schools. Further, our intention with this study was not to compare the relative merits of the varying operationalizations of student wellbeing nor to test these within complete mental health applications in practice. Rather, we took narrower aim at the defensibility of the particular measurement model proposed by the Student Subjective Wellbeing Questionnaire (SSWQ), a school-focused instrument developed chiefly for the purpose of facilitating complete mental health screening when used in conjunction with a problem- or distress-oriented screener (Renshaw et al., 2015) and which could be useful for operationalizing PP, PYD, or SEL outcomes within a broader SMH framework. We submit that the value of context-specific measures like the SSWQ is the inherent specificity of information they are 
assessing -in the SSWQ's case, positive behaviors, emotions, and attitudes toward experiences at school. It follows then that the SSWQ may be preferrable for school-based screening applications compared to general measures (e.g., Positive and Negative Affect Schedule for Children [Laurent et al., 1999]; MSLSS [Huebner, 1994]) due the more direct relevance of the item content to youth wellbeing specifically within the school context than in their lives more broadly, justifying continued study of the measure for both applied and research purposes.

\section{Previous Research with the Student Subjective Wellbeing Questionnaire}

The SSWQ is a 16-item, self-report measure for gauging youth perceptions of their schoolspecific subjective wellbeing. The instrument's item content relates to four dimensions of student wellbeing (SW) - joy of learning (JL; 4 items), school connectedness (SC; 4 items), educational purpose (EP; 4 items), and academic efficacy (AE; 4 items) -that contribute to overall positive psychological functioning at school (Renshaw et al., 2015). Past research provides evidence for deriving scores at the subscale level-representing JL, SC, EP, and AE-and at the global composite level-representing general subjective wellbeing (gSW). Yet previous research appears to have some relative methodological weaknesses, suggesting the need for updated and more contemporary analytic approaches and careful reconsideration of the factor structure.

To begin with, the dimensionality of the SSWQ was originally established using exploratory factor analyses (EFA) on responses from a sample of majority Black students $(N=500)$ in Grades $6-8$ (Renshaw et al., 2015). This EFA used a maximum-likelihood extraction method with a promax rotation, with results yielding four factors with theoretically consistent item content indicated by relatively strong loadings $(\mathrm{AE} \lambda$ range $=.61-.75 ; \mathrm{SC} \lambda$ range $=.52-.79 ; \mathrm{J} \mathrm{L} \lambda$ range $=.52-.74 ; \mathrm{EP} \lambda$ range $=.51-.68)$ that accounted for approximately $58 \%$ of the total variance. The internal consistency reliability of each of the subscales and the total scale (a sum of all subscales) were adequate $(\alpha>.70)$ and the observed correlations ( $r$ ) among subscale scores ranged from .30-.60. Although these results supported a measurement model that was consistent with the authors' conceptual model, the analytic approach was 
limited in two ways. Specifically, the EFA failed to account for the ordinal nature of the data (i.e., 4-point rating scale response options, representing relative frequency of experiencing wellbeing from "almost never" to "almost always") and also failed to investigate the purported higher-order nature of the conceptual model (i.e., the AE, SC, JL, and EP factors were assumed to represent a higher-order gSW factor, yet the EFA only investigated a correlated-factors model). The internal consistency reliability and inter-factor correlations were also only explored at the observed level with latent-level performance left uninvestigated.

Subsequent studies of the SSWQ generated additional evidence for a correlated-factors structure and established the viability of a higher-order model, where each of the four first-order factors (i.e., JL, SC, EP, AE) are specified as indicators of one higher-order factor (i.e., gSW; Arslan \& Renshaw, 2018; Renshaw \& Chenier, 2018). These studies all yielded evidence to support good global data-model fit, adequate to strong loadings across factors, and strong latent internal consistency reliability $(H)$ for both the first-order and second-order factors. However, similar to the original development study described above (Renshaw et al., 2015), these follow-up studies employed less optimal psychometric analyses. Specifically, these later studies also failed to appropriately account for the ordinal nature of the rating scale data when factoring the measurement model (i.e., using the maximum likelihood estimator instead of an estimator designed for categorical responses). Furthermore, data-model fit was only evaluated via global indices without attention to local fit and the internal consistency reliability estimates did not account for the higher-order nature of the measurement model.

\section{The Present Study: Fine-Tuning the SSWQ}

Given the limitations of previous SSWQ work, our primary aim in the present study was to refine the interpretation and use of scores from the SSWQ by testing multiple approaches to specifying the full, 16-item SSWQ measurement model using updated analytic methods. We started by conducting a more appropriately formulated EFA that accounted for the higher-order nature of the SSWQ's conceptual model. Following, we evaluated correlated-factors and higher-order models supported in 
previous studies while accounting for the ordinal nature of the data, and then extended the evaluation

to include bifactor and unidimensional models, which had yet to be investigated. We also conducted a more robust and thoroughgoing evaluation of factor reliability that matched the structure of the preferred measurement models. Finally, we investigated the strength of correlation between factor scores and observed scores, which also had not previously been done. Taken together, findings from these several models were intended to provide a more nuanced evidence-based understanding of how the SSWQ's factor structure could most usefully be conceptualized for complete mental health measurement in schools.

Consistent with previous SSWQ research, we anticipated the correlated-factors and higher-order models would show acceptable data-model fit. The bifactor model specification was expected to result in superior fit, given that the method imposes few constraints (Bornovalova et al., 2020). More importantly, the bifactor approach allowed for partitioning sources of variance between the general factor (gSW) and group factors (JL, SC, AE, and EP) independent of each other which allows for additional insights. Parsing the variance in this way is helpful in determining how uniquely reliable factor scores are from one another (Rodriguez et al., 2016b; Canivez, 2016). Yet it is important to note that interpretation of bifactor models can be challenging and measures of data-model fit may be biased to prefer bifactor models over competing structures often even if fit to data with random patterns (Bonifay et al., 2017). In this context, the bifactor results should not necessarily be thought of as a theoreticallyderived test of a factor structure we believe to actually align with student wellbeing in reality, but one of many useful tools to understand certain aspects of the SSWQ response patterns not yet explored.

We anticipated that the general student wellbeing factor would have the strongest psychometric characteristics (e.g., reliability indices, factor loadings) while the group factors' characteristics would be weaker but still acceptable. The unidimensional model was not expected to fit well given the past evidence for a multidimensional structure but was important for comparison and to enhance interpretation of the bifactor model indices. Finally, we tested the defensibility of a parallel 
unit-weighted (i.e., all items given equal weight) bifactor structure. If this specification showed good data-model fit, it would provide strong empirical support for simple summing of raw item responses to obtain factor scores in latent modeling applications. Correlation $\geq .99$ between parallel unit-weighted and congeneric optimally-weighted scores (i.e., responses weighted based on model characteristics such as item factor loading) would provide additional evidence for score equivalence (McNeish \& Wolf, 2020). To date, score equivalence evidence has not been considered for the SSWQ nor for any other wellbeing metrics used in complete mental health measurement models.

\section{Method}

\section{Participants}

Data were collected in February 2020 at selected high schools partnering with the Character Lab Research Network (CLRN; https://characterlab.org/research-network). The identity of partner schools was blind to the researchers, with data collection facilitated by CLRN staff using a Qualtrics survey protocol developed by the researchers. As a matter of policy, CLRN does not facilitate studies that collect personal health information or that ask questions that would violate the Protection of Pupil Rights Amendment. This project was determined exempt from Institutional Review Board (IRB) oversight via the CLRN's IRB, provided by Advarra (https://www.advarra.com).

The present sample was derived from the CLRN's partner schools within Stratum E (mostly White, high SES, mostly suburban), which represent $21 \%$ of all secondary schools in the United States. This stratified sampling method was developed by CLRN in consultation with their Scientific Advisory Council member Beth Tipton, using the method she developed (Tipton, 2014) and implemented in The Generalizer (https://thegeneralizer.org). A total of 1,020 students in grades 9-12 participated in the online data collection through Qualtrics during a dedicated 25-minute block of in-class time on a school day. The specific sample size obtained was based on CLRN protocols for standard "fully powered" studies and was determined by their methodologists who considered the particular needs of the proposed study. This full sample was divided into randomly split halves resulting in subsample 1 (S1; $N=$ 
529) and subsample 2 (S2; $N=491$ ) used for the exploratory (S1) and confirmatory (S2) phases of analysis respectively.

Categorical demographic information for the participants is presented in Table 1 split by the full sample, S1, and S2. Participants reported a median age of 15 in both subsets (S1: $M=15.5, S D=1.3 ;$ S2: $M=15.6, S D=1.3)$. Median overall GPA for the year through third-quarter data collection in S1 was $86.6 \%(M=83.5 \%, S D=10.5 \%)$ and $87 \%$ in S2 $(M=83.9 \%, S D=10.4 \%)$. All categorical demographic variables apart from self-reported gender and grade-level were based on 495 (S1) and 460 (S2) cases, as information for 65 participants in the full sample was unavailable in the demographics dataset provided by CLRN after primary data collection was complete.

Table 1

Participant Demographic Information

\begin{tabular}{|c|c|c|c|c|c|c|c|c|c|}
\hline & \multicolumn{3}{|c|}{ Full Sample } & \multicolumn{3}{|c|}{ Subsample 1} & \multicolumn{3}{|c|}{ Subsample 2} \\
\hline & $N$ & $n$ & $\%$ & $N$ & $n$ & $\%$ & $N$ & $n$ & $\%$ \\
\hline SR Grade & 1020 & & & 529 & & & 491 & & \\
\hline $9^{\text {th }}$ & & 381 & 37.4 & & 203 & 38.4 & & 178 & 36.3 \\
\hline $10^{\text {th }}$ & & 290 & 28.4 & & 145 & 27.4 & & 145 & 29.5 \\
\hline $11^{\text {th }}$ & & 217 & 21.3 & & 112 & 23.1 & & 94 & 19.1 \\
\hline $12^{\text {th }}$ & & 121 & 11.9 & & 56 & 10.6 & & 65 & 13.2 \\
\hline Not Reported & & 11 & 1.1 & & 2 & 0.3 & & 9 & 1.8 \\
\hline SR Gender & 1020 & & & 529 & & & 491 & & \\
\hline Female & & 539 & 52.8 & & 279 & 52.7 & & 260 & 53.0 \\
\hline Male & & 457 & 44.8 & & 242 & 45.7 & & 215 & 43.8 \\
\hline Other & & 18 & 1.8 & & 7 & 1.3 & & 11 & 2.2 \\
\hline Not Reported & & 6 & 0.6 & & 1 & 0.2 & & 5 & 1.0 \\
\hline Race/Ethnicity & 955 & & & 495 & & & 460 & & \\
\hline Am. Indian & & 5 & 0.5 & & 1 & 0.2 & & 4 & 0.9 \\
\hline Asian & & 79 & 8.3 & & 39 & 7.9 & & 40 & 8.7 \\
\hline Black & & 71 & 7.4 & & 29 & 5.9 & & 42 & 9.1 \\
\hline Hispanic & & 382 & 40.0 & & 205 & 41.4 & & 177 & 38.5 \\
\hline Pacific Islander & & 6 & 0.6 & & 3 & 0.6 & & 3 & 0.7 \\
\hline White & & 779 & 81.6 & & 416 & 84.0 & & 363 & 78.9 \\
\hline Multiracial & & 15 & 1.6 & & 7 & 1.4 & & 8 & 1.7 \\
\hline FRPL & 955 & 224 & 23.5 & 495 & 119 & 24.0 & 460 & 105 & 22.8 \\
\hline ELL & 955 & 95 & 9.9 & 495 & 46 & 9.3 & 460 & 49 & 10.7 \\
\hline SPED & 955 & 146 & 15.3 & 495 & 72 & 14.5 & 460 & 74 & 16.1 \\
\hline Retained & 955 & 3 & 0.3 & 495 & 2 & 0.4 & 460 & 1 & 0.2 \\
\hline Home Language & 955 & & & 495 & & & 460 & & \\
\hline English & & 597 & 62.5 & & 308 & 62.2 & & 289 & 62.8 \\
\hline Spanish & & 163 & 17.1 & & 81 & 16.4 & & 82 & 17.8 \\
\hline Other & & 195 & 20.4 & & 106 & 21.4 & & 89 & 19.3 \\
\hline
\end{tabular}


Note. $N$ indicates the number of cases available for descriptive analysis from the total sample. SR = selfreported; FRPL = Free or reduced price lunch; ELL = English language learner; SPED = Receiving special education services. Retained indicates if the student is repeating the current grade. Participants were allowed to select multiple racial/ethnic identities resulting in the cumulative $n$ exceeding the total respondents.

\section{Measures}

Scores derived from the SSWQ were the only measures in this study. As described above, the SSWQ is a 16-item self-report rating scale for measuring youths' perceptions of their positive psychological functioning at school. All items are arranged along a four-point response scale for evaluating relative frequency of experiences $(1=$ almost never, $2=$ sometimes, $3=$ often, $4=$ almost always). Previous studies suggested the SSWQ can be used for gauging general student wellbeing as well as four subdomains of school-specific wellbeing: joy of learning (JL), school connectedness (SC), educational purpose (EP), and academic efficacy (AE). A copy of the SSWQ and a brief user guide have been made publicly available on the Open Science Framework: https://osf.io/48av7.

\section{Data Analyses}

All analyses were conducted in the R statistical environment (ver. 4.1.2; R Core Team, 2021) using functions from base $\mathrm{R}$ and the following packages: tidyverse (ver. 1.3.1; Wickham et al., 2019), psych (ver. 2.1.9; Revelle, 2021), EFAtools (ver. 0.3.1; Steiner \& Greider, 2020), lavaan (ver. 0.6-9; Rosseel, 2012), and BifactorIndicesCalculator (ver. 0.2.2; Dueber, 2021). Data were cleaned and screened before conducting the primary analyses. Visual inspection of item-level plots and descriptive summaries indicated the absence of substantially aberrant outlying data among all 16 SSWQ items. No missing data were found among the SSWQ item responses in either S1 or S2.

\section{Factor Models}

The latent structure of the SSWQ was probed using a series of factor models starting with exploratory factor analysis (EFA) using data from S1. The optimal number of factors to retain was determined in a multicomponent fashion through parallel analysis simulation and visual inspection of 
eigenvalue scree plots (Garrido et al., 2013). Models were evaluated by inspecting the pattern matrix loadings adjusted with Kaiser normalization and a promax rotation to aid in interpretability. Models were checked for sufficiently large pattern matrix loadings $(\lambda>.30)$ among items that make theoretical sense to load together on the same factor and no large cross-loadings $(\lambda<.30)$ in the interest of preserving simple structure. Items not meeting these criteria were considered for removal. All parallel analyses and EFA solutions were estimated based on polychoric correlation matrices better suited to the ordinal data (Holgado-Tello et al., 2010). Once a preferred factor solution was found, a Schmid-Leiman transformation was applied to that model in order to evaluate the relative variance accounted for by each first-order factor in light of a second-order general factor (Schmid \& Leiman, 1957).

In the next stage, data from S2 were used to conduct five confirmatory factor analyses (CFA) to evaluate the defensibility of a priori specified factor structures based on the conclusions from the EFA results and findings from previous studies with the SSWQ. The unidimensional model specified all items as indicators of a single general Student Wellbeing factor (gSW). The correlated-factors model specified the SSWQ items as indicators of each of their respective group factors ( 4 items per factor) and factor scores were allowed to freely covary with one another. The higher-order model extended the correlated-factors model by specifying each of the first-order factors as indicators of a second-order gSW factor. The Schmid-Leiman transformation was applied to this model as well. The bifactor models specified all SSWQ items as first-order indicators of both their respective group factor, orthogonal with the other factors as well as with the gSW. Finally, in addition to the congeneric bifactor model, a parallel bifactor model was also fit, constraining all item loadings and residual variances to be equal within factors to test the viability of simple sum scoring procedures (McNeish \& Wolf, 2020).

To appropriately account for the ordinal nature of the item responses, a weighted least squares estimator with mean and variance adjustment (WLSMV) was used for all models, along with robust variants of model $\chi^{2}, \mathrm{CFI}, \mathrm{TLI}, \mathrm{RMSEA}$, and SRMR global fit indices. The following interpretive heuristics were used to determine acceptable global fit: $\mathrm{CFI} / \mathrm{TLI} \geq .90$ (adequate) or $\geq .95$ (strong); RMSEA $\leq .08$ 
(adequate) or $\leq .05$ (strong); SRMR $\leq .10$ (adequate) or $\leq .08$ (strong). Local data-model fit was evaluated

by inspection of modification indices and residual correlations from the fitted models, with correlation values $<|.10|$ considered sufficiently small for indicating acceptable local fit (Kline, 2016).

\section{Model-Based Reliability Indices}

A variety of model-based reliability indices were considered as part of evaluating the technical adequacy of derived scores. Omega $(\omega)$ was used as a measure of unit-weighted reliability of scores considered independently and is most similar to traditional Cronbach's alpha though provides more accurate estimates in most situations (McNeish, 2018). Omega hierarchical/hierarchical subscale ( $\left.\omega_{H / H S}\right)$ reflects the amount of unique variance attributable to a general factor alone $\left(\omega_{H}\right)$ or unique variance in a group factor after accounting for the general factor $\left(\omega_{H S}\right)$. Construct replicability $(H)$ was used as a measure of optimally-weighted reliability and reflects how well each set of items represents their latent factor. Factor determinacy $(F D)$ assessed the degree that individual differences in factor scores reflect true differences on the factor (Grice, 2001). $H$ values $\geq .70$ were considered acceptable (Hancock \& Mueller, 2001); $\omega$ and $\omega_{H / H S}$ values $\geq .50$ were considered minimally acceptable and $\geq .75$ preferred (Reise et al., 2013); and $F D$ values $\geq .90$ indicated adequate support for interpreting factor scores (Gorsuch, 1983, as cited in Rodriguez et al., 2016b).

\section{Correlations}

Correlation analyses were conducted to investigate associations among the derived factor scores. This included zero-order correlations among unit-weighted and optimally-weighted scores as well as partial correlations among unit-weighted group factor scores. A regression approach was used to compute optimally-weighted factor scores based on the preferred congeneric bifactor model.

\section{Results}

\section{Factor Models}

\section{Exploratory Models}


16-Item EFA. Preliminary results from the first EFA of all 16 SSWQ items showed strong sampling adequacy $(\mathrm{KMO}=.915)$ and a lack of singularity $\left(\chi^{2}{ }_{(120)}=4,027.21, p<.001\right)$ suggesting these data are appropriate for factor analysis. Results from parallel analysis indicated that a four-factor solution would best characterize the data above chance levels. Constraining a four-factor solution revealed a structure with strong item communalities $\left(h^{2}\right.$ range $\left.=.54-.84\right)$ that accounted for approximately $68 \%$ of total variability in the scores. Inspection of the pattern matrix showed that the four SC items ( $\lambda$ range $=.67-$ .82 ) and four $A E$ items ( $\lambda$ range $=.70-.95)$ loaded strongly on distinct factors with no significant crossloadings, as expected. The four JL items also loaded strongly on a third factor without notable crossloadings ( $\lambda$ range $=.72-.81$ ); however, two EP items also cross-loaded highly on the same factor as the $\mathrm{JL}$ items-SSWQ3 ("I feel like the things I do at school are important"), $\lambda=.52$; SSWQ15 ("I believe the things I learn at school will help me in my life"), $\lambda=.67$. In addition, these two EP items also showed acceptably large loadings with a fourth factor on which the other two EP items loaded (SSWQ7 "I think school matters and should be taken seriously"; SSWQ11 "I feel it is important to do well in my classes"; $\lambda$ range $=.31-.84)$, though these were not as large as the loadings with the $\mathrm{JL}$ items.

A three-factor model was also fit, producing similar results but with the elimination of factor four where the EP items SSWQ7 and SSWQ11 loaded by themselves. In this three factor solution, these two EP items loaded with the AE items. Considering these results in light of the evidence and potential theoretical disconnect between the two subsets of EP items - two of which related to belief in the benefits of school (and loaded with the JL items) and the other two related to the importance of performing one's best in school (and loaded with the AE items)-we chose to remove all four EP items and instead consider the defensibility of 12 -item factor solutions in subsequent analyses.

12-Item EFA. The data for the remaining 12 items were characterized again by acceptable sampling adequacy $(\mathrm{KMO}=.892)$ and lack of singularity $\left(\chi^{2}{ }_{(66)}=2,977.23, p<.001\right)$. Scree plot inspection and parallel analysis suggested that three factors meaningfully accounted for the item responses above chance levels. The derived three-factor solution resulted in predominantly strong communalities $\left(h^{2}\right.$ 
range $=.47-.86)$ and accounted for approximately $66 \%$ of total variability in the scores. Inspection of the pattern matrix showed that the three clusters of $\mathrm{JL}(\lambda$ range $=.71-.84), \mathrm{SC}(\lambda$ range $=.64-.85)$, and $\mathrm{AE}(\lambda$ range $=.78-.96)$ items each loaded strongly to distinct factors with no notable cross-loadings.

Having found a defensible factor solution, the Schmid-Leiman transformation was then applied to investigate the model incorporating second-order loadings and to partition the variance in factors. The second-order general factor loadings were strong across all items $(\lambda$ range $=.56-.71)$ and loadings for the item clusters in the three group factors remained similarly strong as in the untransformed model. Inspection of model-based indices showed that the general factor accounted for the most variance in scores and was the only one of the four factors to show acceptable score reliability $\left(\omega_{H}=.74\right)$ after controlling for the unique variance captured by the other factors ( $\omega_{H S}$ range $=.29-.53$ ). See Table 2 for full results.

Table 2 Principal Axis Factor Analysis Results with Schmid-Leiman Transformation for the Preferred 3Factor SSWQ Solution

\begin{tabular}{|c|c|c|c|c|c|}
\hline Item & gSW & $\mathrm{JL} \lambda$ & AE $\lambda$ & $\mathrm{SC \lambda}$ & $h^{2}$ \\
\hline 1. Excited & .563 & .531 & -.005 & -.063 & .602 \\
\hline 13. Нарру & .711 & .489 & .006 & .076 & .750 \\
\hline 9. Enjoy & .572 & .470 & -.005 & -.000 & .548 \\
\hline 5. Interested & .623 & .450 & .008 & .046 & .592 \\
\hline 16. Good grades & .578 & -.020 & .728 & -.022 & .864 \\
\hline 4. Successful & .564 & -.031 & .680 & .007 & .781 \\
\hline 12. Do well & .584 & -.003 & .676 & -.000 & .798 \\
\hline 8. Good work & .632 & .076 & .587 & .021 & .750 \\
\hline 10. Care & .655 & -.015 & -.013 & .502 & .681 \\
\hline 2. Belong & .623 & .026 & -.014 & .442 & .584 \\
\hline 6. Be myself & .560 & .003 & -.027 & .424 & .493 \\
\hline 14. Respect & .569 & -.004 & .065 & .379 & .472 \\
\hline Proportion Total Var & .37 & .08 & .15 & .07 & \\
\hline Proportion Common Var & .55 & .12 & .23 & .10 & \\
\hline$\omega$ & .94 & .87 & .94 & .83 & \\
\hline $\boldsymbol{\omega}_{H / H S}$ & .74 & .33 & .53 & .29 & \\
\hline
\end{tabular}

Note. SSWQ = Student Subjective Wellbeing Questionnaire; gSW = General subjective wellbeing second-order factor; $\mathrm{JL}=$ Joy of Learning; $\mathrm{AE}=$ Academic Efficacy; $\mathrm{SC}=$ School Connectedness; $\lambda$ $=$ Standardized pattern matrix factor loadings; $h^{2}=$ Item communalities; Var = Variance; IECV = Item explained common variance; $\omega=$ Omega; $\omega_{H / H S}=$ Omega hierarchical/hierarchical subscale. Bolded values indicate standardized factor loadings $>.30$ or minimally acceptable $\omega / \omega_{H / H S}$. Criteria for acceptable reliability: $\omega, \omega_{H / H S} \geq .50 / .75$. 


\section{Confirmatory Models}

Data-model fit indices for all tested CFA models are reported in Table 3 and discussed next.

Table 3

Confirmatory Factor Analysis Model Fit Indices

\begin{tabular}{lccccc}
\hline \multicolumn{1}{c}{ Model } & $\boldsymbol{\chi}^{\mathbf{2}}$ (df) & CFI & TLI & RMSEA $[90 \%$ Cl] & SRMR \\
\hline Unidimensional & $1,300.01(54)$ & .837 & .800 & $.217[.207, .227]$ & .147 \\
Cor Factors & $156.85(51)$ & .986 & .982 & $.065[.054, .077]$ & .039 \\
Higher Order & $156.85(51)$ & .986 & .980 & $.065[.054, .077]$ & .039 \\
Bifactor & $80.59(42)$ & .995 & .992 & $.043[.029, .057]$ & .023 \\
Parallel BF & $193.72(59)$ & .982 & .980 & $.068[.058, .079]$ & .061 \\
\hline
\end{tabular}

Note. Cor $=$ Correlated/Oblique factors model; BF = Bifactor.

Unidimensional Structure. The 12-item unidimensional model had relatively strong factor loadings ( $\lambda$ range $=.63-.84$ ) but was poorly fit to the data based on global indices. In addition, inspection of residual correlations showed 45 item pairs with correlations $>|.10|$, indicating very poor local fit, as expected given the prior evidence for a multidimensional structure.

Correlated-Factors Structure. The correlated-factors model had consistently strong loadings across the three specified factors ( $\mathrm{JL} \lambda$ range $=.73-.85 ; \mathrm{SC} \lambda$ range $=.76-.83 ; \mathrm{AE} \lambda$ range $=.87-.89$ ) and showed much improved global data-model fit compared to the unidimensional structure. Standardized covariances between factors were medium to large in magnitude ( $\varphi$ range $=.48-.70$ ), suggesting potential influence of a general factor left unaccounted for in the present model. Inspection of residual correlations now revealed only two item pairs with correlations slightly greater than |.10| -SSWQ8 ("I do good work in school") with SSWQ5 ("I am really interested in the things I am doing at school") and SSWQ9 ("I enjoy working on class projects and assignments"), supporting significantly improved local fit. Summaries and plots of modification indices suggested respecifying two of the AE items (SSWQ8 and SSWQ16) to the JL factor would result in the greatest improvement in fit. However, these changes were not made due to insufficient theoretical justification for measurement purposes.

Higher-Order Structure. Compared to the correlated-factors model, the higher-order model produced practically identical indices of global data-model fit, first-order factor loadings, and residual 
correlations among items. However, this structure also showed large second-order loading paths with each of the first-order factors, most significantly with the JL factor $(\mathrm{JL} \gamma=.94 ; \mathrm{SC} \gamma=.74 ; \mathrm{AE} \gamma=.64)$.

As done with the 12-item EFA model, a Schmid-Leiman transformation was applied to partition the variance of each factor in this structure considering the influence of the transformed second-order solution. This resulted in the same conclusion as results from the 12-item EFA, with the global secondorder factor accounting for the majority of variance among the item responses and being the only factor with preferably large unique reliability $\left(\omega_{H}=.77\right)$ when considered independently from the first-order factors $\left(\omega_{H S}\right.$ range $\left.=.11-.55\right)$.

Bifactor Structure. The 12 SSWQ items were then specified in a congeneric bifactor structure. Full model results are reported in Table 4. Global indices of data-model fit were uniformly strong as was the magnitude of factor loadings for the general factor $(\lambda$ range $=.46-.82)$, SC group factor $(\lambda$ range $=$ $.47-.60)$, and AE group factor ( $\lambda$ range $=.58-.80)$, while JL group factor loadings were notably weaker $(\lambda$ range $=.19-.40)$. Inspection of residual correlations showed all associations between item pairs to be $<|.10|$, indicating strong local data-model fit.

Consistent with our prior results, bifactor model-based reliability estimates again supported the dominance of the general factor $\left(\omega_{H}=.77\right)$ over any of the group factors after accounting for variance associated with the other factors ( $\omega_{H S}$ range $\left.=.11-.55\right)$. Factor replicability $(H)$ and factor determinacy $(F D)$ indices were both strong for gSW in this model as well as for the AE group factor, but less than adequate for the JL and SC group factors.

Considering relative parameter bias (RPB), JL and SC items had relatively small values (RPB range $=.01-.20$ ) while values for AE items were considerably larger (RPB range $=.27-.80$ ). The average relative parameter bias for the 12-item, optimally-weighted SSWQ structure was .23, which exceeded the suggested threshold of approximately $.10-.15$ to consider scores essentially unidimensional for latent modeling applications (Muthén et al., 1987). 
Table 4

SSWQ 12-item Congeneric Unidimensional and Bifactor Model Results

\begin{tabular}{|c|c|c|c|c|c|c|c|c|c|c|c|c|c|}
\hline & \multirow{2}{*}{\multicolumn{2}{|c|}{$\begin{array}{c}\text { Uni Model } \\
\text { gSW }\end{array}$}} & \multicolumn{9}{|c|}{ Bifactor Model } & \multirow[b]{3}{*}{ IECV } & \multirow[b]{3}{*}{ RPB } \\
\hline & & & \multicolumn{2}{|l|}{ gSW } & \multicolumn{2}{|l|}{ JL } & \multicolumn{2}{|l|}{ SC } & \multicolumn{2}{|l|}{$\mathrm{AE}$} & \multirow[b]{2}{*}{ Var } & & \\
\hline & Est (SE) & Std & Est (SE) & Std & Est (SE) & Std & Est (SE) & Std & Est (SE) & Std & & & \\
\hline 1. Excited & 1.00 & .68 & 1.00 & .69 & 1.00 & .40 & & & & & .36 & .75 & .01 \\
\hline 5. Interested & $1.10(.04)$ & .75 & $1.13(.06)$ & .78 & $0.72(.31)$ & .29 & & & & & .30 & .88 & .04 \\
\hline 9. Enjoy & $0.94(.04)$ & .64 & $0.98(.06)$ & .68 & $0.67(.32)$ & .27 & & & & & .47 & .86 & .06 \\
\hline 13. Нарру & $1.11(.04)$ & .76 & $1.19(.07)$ & .82 & $0.48(.27)$ & .19 & & & & & .29 & .95 & .08 \\
\hline 2. Belong & $1.02(.05)$ & .70 & $0.91(.09)$ & .63 & & & 1.00 & .50 & & & .36 & .62 & .10 \\
\hline 6. Be myself & $0.92(.05)$ & .63 & $0.76(.08)$ & .53 & & & $1.21(.12)$ & .60 & & & .36 & .43 & .20 \\
\hline 10. Care & $0.98(.05)$ & .67 & $0.84(.09)$ & .58 & & & $1.12(.10)$ & .56 & & & .35 & .52 & .14 \\
\hline 14. Respect & $0.93(.05)$ & .66 & $0.83(.09)$ & .58 & & & $0.94(.09)$ & .47 & & & .45 & .60 & .10 \\
\hline 4. Successful & $1.19(.04)$ & .81 & $0.81(.08)$ & .56 & & & & & 1.00 & .65 & .26 & .43 & .44 \\
\hline 8. Good work & $1.23(.04)$ & .84 & $0.96(.09)$ & .67 & & & & & $0.89(.05)$ & .58 & .23 & .57 & .26 \\
\hline 12. Do well & $1.20(.05)$ & .82 & $0.81(.08)$ & .56 & & & & & $1.05(.05)$ & .68 & .23 & .40 & .47 \\
\hline 16. Good grades & $1.20(.05)$ & .82 & $0.66(.08)$ & .46 & & & & & $1.23(.06)$ & .80 & .16 & .24 & .80 \\
\hline Latent Factor Var & $.47(.03)$ & 1.00 & $.48(.06)$ & 1.00 & $.16(.09)$ & 1.00 & $.25(.04)$ & 1.00 & $.42(.04)$ & 1.00 & & & \\
\hline$\omega$ & .93 & & .95 & & .88 & & .87 & & .93 & & & & \\
\hline$\omega_{\mathrm{H} / \mathrm{HS}}$ & .93 & & .77 & & .11 & & .40 & & .55 & & & & \\
\hline$H$ & .94 & & .90 & & .29 & & .62 & & .79 & & & & \\
\hline$F D$ & .97 & & .92 & & .52 & & .81 & & .91 & & & & \\
\hline
\end{tabular}

Note. SSWQ = Student Subjective Wellbeing Questionnaire; gSW = General subjective wellbeing; JL = Joy of Learning; SC = School Connectedness; $\mathrm{AE}=$ Academic Efficacy. Uni = Unidimensional; Est = Unstandardized estimate; SE = Standard error; Std = Standardized estimate; Var = Variance; IECV = Item explained common variance; RPB = Relative parameter bias; $\omega=$ Omega; $\omega_{H / H S}=$ Omega hierarchical/hierarchical subscale; $H=$ Construct replicability; $F D=$ Factor determinacy.

Bolded values indicate acceptable reliability for each index.

Criteria for acceptable reliability: $\omega, \omega_{H / H S} \geq .50 / .75 ; H \geq .70 ; F D \geq .90$. 
Parallel Bifactor Structure. Finally, the same 12-item bifactor model was fit constraining all loadings and residual variances to equality within factors, to test the tenability of a parallel model that reflects the assumptions made when producing unit-weighted (or simple sum) scores from each factor in a latent variable modeling framework. Global fit indices for this unit-weighted model were acceptable although nine residual correlations were $>|.10|$, primarily involving items between the $\mathrm{JL}$ and $A E$ factors, providing mixed support for simple sum scoring of raw item ratings based on latent structure. Reliability indices for this unit-weighted model were highly similar to those from the congeneric model, again providing support for the adequate reliability of gSW $\left(\omega_{H}=.77\right)$ but little support for the unique reliability of the three group factors independently ( $\omega_{H S}$ range $=.30-.45$ ).

\section{Correlation Analyses}

Descriptive statistics for item composites for each of the 12-item SSWQ factors of interest in this study (i.e., gSW, JL, SC, and AE, but excludes EP) are found in Table 5. All correlations among these scores based on data from S2 are presented in Table 6. Zero-order correlations among the unit-weighted scores were moderate to large in magnitude $(r$ range $=.39-.84)$. Partial correlations among the three unit-weighted group factor scores were small to moderate $\left(r_{\mathrm{p}}\right.$ range $\left.=.16-.46\right)$. Zero-order correlations between unit- and optimally-weighted scores were large, with gSW showing the strongest relation $(r=$ $.95)$; however, these values fell below the suggested minimum correlation of .99 to support equivalence. For comparisons with past research, the correlation between the preferred 12 -item set and the full 16item set was extremely strong $(r[95 \% \mathrm{Cl}]=.98[.97, .98])$ in both $\mathrm{S} 1$ and $\mathrm{S} 2$. 
Table 5

Descriptive Statistics of the 12-Item SSWQ Scores

\begin{tabular}{cccccccccc}
\hline Score & Subset & AIS (SE) & $\boldsymbol{M}$ & $\boldsymbol{S D}$ & Med & Min & Max & Skew & Kurt \\
\hline \multirow{2}{*}{ gSW 12 } & S1 & $2.63(.03)$ & 31.58 & 7.25 & 32 & 12 & 48 & -0.10 & -0.12 \\
& S2 & $2.64(.03)$ & 31.66 & 7.37 & 31 & 12 & 48 & -0.05 & -0.26 \\
JL & S1 & $2.29(.03)$ & 9.16 & 2.88 & 9 & 4 & 16 & 0.44 & -0.32 \\
& S2 & $2.28(.03)$ & 9.14 & 2.97 & 9 & 4 & 16 & 0.41 & -0.37 \\
SC & S1 & $2.62(.03)$ & 10.49 & 2.97 & 11 & 4 & 16 & -0.12 & -0.55 \\
& S2 & $2.63(.03)$ & 10.54 & 3.03 & 10 & 4 & 16 & -0.10 & -0.70 \\
AE & S1 & $2.98(.03)$ & 11.93 & 3.20 & 12 & 4 & 16 & -0.51 & -0.50 \\
& S2 & $2.99(.04)$ & 11.98 & 3.12 & 12 & 4 & 16 & -0.42 & -0.66 \\
\hline
\end{tabular}

Note. SSWQ = Student Subjective Wellbeing Questionnaire; gSW $12=$ General subjective wellbeing score from the 12-item model; $\mathrm{JL}=$ Joy of Learning; SC = School Connectedness; $\mathrm{AE}=$ Academic Efficacy; $A I S=$ Average item score; $S E=$ Standard error; $M=$ Mean; $S D=$ Standard deviation; Med = Median; Min = Minimum; Max = Maximum; Skew = Skewness; Kurt = Kurtosis.

Table 6

Zero-Order and Partial Correlations with 95\% Confidence Intervals Among SSWQ Scores

\begin{tabular}{ccccc} 
& gSW 12 & $\mathrm{JL}$ & $\mathrm{SC}$ & $\mathrm{AE}$ \\
\cline { 2 - 5 } gSW 12 & $.95[.94, .96]$ & & & \\
$\mathrm{JL}$ & $.84[.81, .86]$ & $.70[.65, .74]$ & $.46[.38, .52]$ & $.36[.28, .43]$ \\
SC & $.80[.77, .83]$ & $.56[.49, .62]$ & $.80[.77, .83]$ & $.16[.08, .25]$ \\
AE & $.78[.75, .81]$ & $.49[.42, .56]$ & $.39[.31, .46]$ & $.82[.79, .85]$
\end{tabular}

Note. gSW 12= General subjective wellbeing score from the 12-item model; $\mathrm{JL}=$ Joy of Learning; SC $=$ School Connectedness; $\mathrm{AE}=$ Academic Efficacy.

Zero-order correlations among total scores are presented below the diagonal and total score partial correlations are presented above the diagonal. Zero-order correlations between unit- and optimallyweighted factor scores from the 12-item bifactor model are in bold on the diagonal.

All correlations were significant at Holm-Bonferroni adjusted $p<.01$.

\section{Discussion}

These results lead to several conclusions and implications for interpretation of SSWQ scores.

The biggest finding was that the four items originally conceptualized as adhering to the EP factor did not load together as expected. Instead, two EP items-related to (1) belief in the importance of school and (2) that things learned at school will be helpful in life-loaded with the JL items, while the other two EP items - related to (1) thinking school matters and should be taken seriously and (2) believing it is important to do well in classes-loaded together on a distinct factor. However, all other items showed empirical coherence as expected with the other three specific factors represented in the SSWQ's 
conceptual model (JL, SC, and $\mathrm{AE}$ ), which ultimately led to the retention of a reduced 12 -item set that excluded the EP items.

Consistent with our expectations, the correlated-factors and higher-order models met our standards of acceptable fit on nearly all indices, as they had in previous studies (e.g., Renshaw, 2015; Arslan \& Renshaw, 2018), while the bifactor model showed the best overall data-model fit. It is noteworthy, however, that all confirmatory structures lacked the EP items that were retained in previous work. When using a bifactor approach, the meaning of the general factor is directly linked to which items are included-thus, removing the four EP items fundamentally changed the interpretation of gSW compared to the full, 16-item version of the SSWQ. Nonetheless, extremely strong correlations between the 12- and 16-item gSW scores $(r=.98)$ in both data subsets suggests that there remains a large amount of overlapping variance $\left(r^{2}=96 \%\right)$ between the original and revised models. Rather than using these results as a basis to discard EP as an important theoretical component to measuring student subjective wellbeing, we instead encourage future researchers to carefully reconsider the item content of this factor/subscale and test whether or not there is a better way to represent EP (and/or the other specific constructs) in the SSWQ measurement model.

Our results also suggest that the gSW scores have many strong reliability characteristics, supporting their use in distinguishing individual differences in self-reported general student wellbeing. However, the SSWQ subscale scores showed mixed (for AE) to no (for JL, SC, EP) supporting evidence for practically meaningful reliability independent of gSW. Though contrary to our hypothesis, other methodological research with bifactor models suggests that the reliability of subscale scores in a wide range of multidimensional measures is frequently inadequate compared to scores from their general factor (see Rodriguez et al., 2016a). As a result, subscale scores may often be misinterpreted as independent representations of their respective constructs, when they are better interpreted interdependently as representative facets of the broader general factor. At the same time, the large discrepancy between the reliability estimates of sum scores when each factor was evaluated in isolation 
$(\omega)$ compared to the hierarchical estimates $\left(\omega_{H / H S}\right)$, along with the high average relative parameter bias, is noteworthy and suggests unignorable multidimensionality.

\section{Recommendations for Use}

Synthesizing these findings, we propose two sets of recommendations for using SSWQ scores in latent variable research and school-based practice contexts, respectively. First, given the evident multidimensionality, future research with the SSWQ that is concerned with measuring the latent variable of student subjective wellbeing should consider using optimally-weighted factor scores drawn from the specified measurement model (bifactor or otherwise) for secondary analyses with other variables of interest (e.g., as covariates or predicted outcomes). Alternatively, relations among variables could be modeled simultaneously, such as in a latent path structural equation modeling framework that includes a full specification of the multidimensional SSWQ latent structure (see McNeish \& Wolf, 2020). Either way, optimal latent variable analyses should account for the SSWQ's multidimensionality to avoid the relative imprecision of using simple sum scores in these contexts (which is admittedly minimal in this case). This recommendation has particular relevance to research regarding complete mental health screening models, which has yet to use this modeling approach.

Second, for applied use in school practice, it would be ideal to fit novel SSWQ data gathered from universal screening to the preferred factor structure to derive optimally-weighted student scores for local use. This could be done by a professional researcher or data analyst working in partnership with the school or district and would increase confidence in the local utility of scores (see Davidson et al. [2018] for a demonstration of such a continuous measure improvement approach through a researchpractice partnership), especially for high-stakes decision making. While using optimally-weighted scores from local data would provide the richest and least biased information, the practical barriers to obtaining these scores will be significant for schools without strong ties to professionals with the appropriate technical skill set. At the same time, the results of this study do provide sufficient evidence to support raw score summing procedures for applied use, especially when making relatively lower- 
stakes decisions, such as determining need for more targeted assessment following Tier I screening in a multigated screening paradigm, which is likely sufficient to meet most schools' needs. That said, for both research and practice, gSW scores should be the only scores used for primary analyses or identification of individual differences in student subjective wellbeing, particularly for the purposes of risk classification, as is common in applications of complete mental health screening (e.g., Moore et al., 2015).

Though our findings suggest that scores from the AE subscale could possibly be interpreted and used independently as well, we recommend this only with considerable caution and always secondary to the interpretation of gSW. Therefore, scores from the JL, SC, or EP subscales should not be substantively interpreted (if interpreted at all) unless future studies identify an alternative set of items that considerably improve their unique psychometric qualities. We acknowledge that subscales might have interpretive worth if used qualitatively after interpretation of gSW. Though this approach would likely only be incrementally useful with accompanying item-level analyses when considering individual students or, better yet, with the incorporation of more specifically targeted measures to clarify particular assessment aims. More work is therefore needed to test particular outcomes from score interpretations before firm recommendations can be made. At the same time, we argue that the subscale scores do not necessarily need to have incremental value over and above the general score for use of the SSWQ to be worthwhile as a screener. In our view, identifying and investigating the relative contributions of the group factors in explaining response variance is useful in and of itself as a means to establish construct representation of the gSW factor and not necessarily to ensure that all modeled factors can be interpreted uniquely and reliably from the others. Strong justification for interpreting all factors in the SSWQ's measurement model would be a desirable feature if the evidence supported it, but it is not, in our opinion, a critical feature for the purposes of an intentionally brief screening measure.

\section{Constraints to Generality and Future Directions}


While we believe this study offers a meaningful contribution toward understanding student

subjective wellbeing measurement for school-based mental health screening via the SSWQ, the results should be viewed in light of several limitations. Although data were analyzed from a relatively large sample of participants, demographic representativeness remains limited in terms of grade-level (only grades 9-12), racial/ethnic identity (predominantly white and Hispanic), socioeconomic status (relatively high), and locale (mostly suburban). Though we do not expect great differences between these groups, future replication studies should make efforts to sample from more diverse populations, with a greater variety of demographic characteristics, to enhance generalizability of findings. Earlier development work with the SSWQ used samples of youth from a broader range of grade-levels (i.e., grades 5-12) and racial/ethnic identity, but future samples should aim to build on the more sophisticated methods of the current study and sample widely again from students of many ages who are also capable of selfreporting as well as students from minoritized and underrepresented populations.

Future work should also focus on testing additional or edited item content to see if the SSWQ's group factors can be measured in a more uniquely reliable and theoretically coherent way, particularly for the educational purpose construct, which was ultimately discarded in its current form based on our analyses. If and when the evidence for interpretation of a preferred SSWQ factor structure stabilizes, the logical next step is to conduct careful measurement invariance studies to compare the consistency with which the structure holds among youth of different grade-levels, genders, and racial/ethnic identities. Relatedly, this study only used a cross-sectional sample and a richer understanding of how well the SSWQ's structure holds over time would come from conducting longitudinal studies with students across and between school years.

Our study focused on rigorous and in-depth analysis of the SSWQ factor structure itself but relations with other theoretically meaningful variables were beyond our intended scope, limiting the range of validity related conclusions that can be drawn from these findings. Future studies can advance this line of work by comparing the relative worth of the SSWQ to other measures of youth wellbeing (or 
related but distinct constructs such as adaptive skills) in their differential ability to estimate current or

future student risk, considering practical indices of diagnostic accuracy (e.g., positive/negative predictive value, sensitivity/specificity) in relation to valued educational outcomes (e.g., academic achievement, attendance/truancy, school dropout, disciplinary infractions). Considering the SSWQ's scores in relation to a wide variety of variables important to school functioning will help strengthen substantive claims of validity though nomological networks in addition to thoughtful consideration for practical consequences of score use (see Kane, 2013; Messick, 1994). Given that the SSWQ is intended as only half of a complete mental health screening endeavor, investigations of its co-use along with problem/distress-oriented screeners is another critical area of future study. In addition, using alternative analytic frameworks than the ones used here, such as multidimensional item response theory, would provide more specific insights into the specific functioning of particular items in relation to their associated factor scores (see Immekus et al., 2019).

\section{Conclusion}

In closing, we suggest this study adds value to the complete mental health measurement literature by further validating and refining the interpretation of scores derived from the SSWQ. Specifically, our findings support the defensibility of a reduced measurement model that, when used to produce composite scores, is best interpreted as a general student wellbeing indicator. Though our particular findings are relatively narrow in scope, we suggest this and related later work may occasion future discussion within the broader field about the number, specificity, and level at which wellbeing constructs are best interpreted and used (cf. Seligman, 2018; Goodman et al., 2018; Linton et al., 2016). Although we tend to think of measurement models of psychopathology and problem behavior as settled, there is more debate and variability in models of wellbeing. It is possible that application of the analytic approaches for factoring and reliability used in this study, which are more rigorous than methods used in many recent wellbeing measurement studies, might yield more nuanced results that 
support rethinking the measurement models of other instruments used for assessing youth subjective wellbeing (see Rodriguez et al., 2016a).

Finally, we wish to re-emphasize that while debates about factor structure and score interpretation are necessary, these concerns are ultimately secondary to understanding the value-added contribution of wellbeing measurement to providing effective mental health supports for youth in schools. We call future researchers to move beyond only evaluating elements of traditional psychometric defensibility, to also investigating the extent to which use of various measurement models actually lead to meaningful improvements in valued student outcomes. 


\section{References}

Appleton, J. J., Christenson, S. L., Kim, D., \& Reschly, A. L. (2006). Measuring cognitive and psychological engagement: Validation of the Student Engagement Instrument. Journal of School Psychology, 44, 427-445. https://doi.org/10.1016/j.jsp.2006.04.002

Arslan, G., \& Renshaw, T. L. (2018). Student subjective wellbeing as a predictor of adolescents' problem behaviors: A comparison of first-order and second-order factor effects. Child Indicators Research, 11, 507-521. https://doi.org/10.1007/s12187-017-9444-0

Bird, J. M., \& Markle, R. S. (2012). Subjective well-being in school environments: Promoting positive youth development through evidence-based assessment and intervention. American Journal of Orthopsychiatry, 82, 61-66. https://doi.org/10.1111/i.1939-0025.2011.01127.x

Bonifay, W., Lane, S. P., \& Reise, S. P. (2017). Three concerns with applying a bifactor model as a structure of psychopathology. Clinical Psychological Science, 5, 184-186.

\section{https://doi.org/10.1177/2167702616657069}

Bornovalova, M. A., Choate, A. M., Fatimah, H., Petersen, K. J., \& Wiernik, B. M. (2020). Appropriate use of bifactor analysis in psychopathology research: Appreciating benefits and limitations. Biological Psychiatry, 88, 18-27. https://doi.org/10.1016/j.biopsych.2020.01.013

Bücker, S., Nuraydin, S., Simonsmeirer, B. A., Schneider, M., \& Luhman, M. (2018). Subjective well-being and academic achievement: A meta-analysis. Journal of Research in Personality, 74, 83-94. https://doi.org/10.1016/i.jrp.2018.02.007

Canivez, G. L. (2016). Bifactor modeling in construct validation of multifactored tests: Implications for understanding multidimensional constructs and test interpretation. In K. Schweizer \& C. DiStefano (Eds.), Principles and methods of test construction: Standards and recent advancements (pp. 247-271). Hogrefe. 
Carr, A., Cullen, K., Keeney, C., Canning, C., Mooney, O., Chinseallaigh, E., \& O’Dowd, A. (2021). Effectiveness of positive psychology interventions: A systematic review and meta-analysis. The Journal of Positive Psychology, 16, 749-769. https://doi.org/10.1080/17439760.2020.1818807

Davidson, L. A., Crowder, M. K., Gordon, R. A., Domitrovich, C. E., Brown, R. \& Hayes, B. (2018). A continuous improvement approach to social and emotional competency measurement. Journal of Applied Developmental Psychology, 55, 93-106.

\section{https://doi.org/10.1016/j.appdev.2017.03.002}

Diener, E. (1984). Subjective well-being. Psychological Bulletin, 95, 542-575.

Diener, E., Emmons, R. A., Larsen, R. J., \& Griffin, S. (1985). The Satisfaction with Life Scale. Journal of Personality Assessment, 49, 71-75. http://internal.psychology.illinois.edu/ ediener/SWLS.html

Dueber, D. (2021). BifactorIndicesCalculator: Bifactor Indices Calculator. R package version 0.2.2. https://CRAN.R-project.org/package=BifactorIndicesCalculator

Garrido, L. E., Abad, F. J., \& Ponsoda, V. (2013). A new look at Horn's parallel analysis with ordinal variables. Psychological Methods, 18, 454-474. https://doi.org/10.1037/a0030005

Goodman, F. R., Disabato, D. J., Kashdan, T. B., \& Kauffman, S. B. (2018). Measuring well-being: A comparison of subjective well-being and PERMA. The Journal of Positive Psychology, 13, 321332. https://doi.org/10.1080/17439760.2017.1388434

Gorsuch, R. L. (1983). Factor analysis (2nd ed.). Hillsdale, NJ: Erlbaum.

Grice, J. W. (2001). Computing and evaluating factor scores. Psychological Methods, 6, 430-450.

\section{http://dx.doi.org/10.1037/1082-989X.6.4.430}

Hancock, G. R., \& Mueller, R. O. (2001). Rethinking construct reliability within latent variable systems. In R. Cudeck, S. du Toit, \& D. Soerbom (Eds.), Structural Equation Modeling: Present und Future-A Festschrift in Honor of Karl Joreskog (p. 195-216). Lincolnwood, IL: Scientific Software International. 
Holgado-Tello, F. P., Chacon-Moscoso, S., Barbero-Garcia, I., \& Vila-Abad, E. (2010). Polychoric versus Pearson correlations in exploratory and confirmatory factor analysis of ordinal variables. Quality \& Quantity, 44, 153-166. https://doi.org/10.1007/s11135-008-9190-y

Huebner, E.S. (1994). Preliminary development and validation of a multidimensional life satisfaction scale for children. Psychological Assessment, 6, 149-158.

Immekus, J. C., Snyder, K. E., \& Ralston, P. A. (2019). Multidimensional item response theory for factor structure assessment in educational psychology research. Frontiers in Education, 4(45). https://doi.org/10.3389/feduc.2019.00045

Kane, M. (2013). The argument-based approach to validation. School Psychology Review, 42, 448-457. https://doi.org/10.1080/02796015.2013.12087465

Kline, R. B. (2016). Principles and practice of structural equation modeling (4th ed.). New York, NY: Guilford Publications, Inc.

Laurent, J., Catanzaro, S. J., Joiner Jr, T. E., Rudolph, K. D., Potter, K. I., Lambert, S., Osborne, L., \& Gathright, T. (1999). A measure of positive and negative affect for children: Scale development and preliminary validation. Psychological Assessment, 11, 326-338.

Lerner, R. M., Almerigi, J. B., Theokas, C., \& Lerner, J. V. (2005). Positive youth development: A view of the issues. The Journal of Early Adolescence, 25, 10-15. https://doi.org/10.1177/0272431604273211

Linton, M., Dieppe, P., \& Medina-Lara, A. (2016). Review of 99 self-report measures for assessing wellbeing in adults: Exploring dimensions of well-being and developments over time. BMJ Open, 6:e010641. http://dx.doi.org/10.1136/bmjopen-2015-010641

McNeish, D. (2018). Thanks coefficient alpha, we'll take it from here. Psychological Methods, 23, 412433. https://doi.org/10.1037/met0000144

McNeish, D., \& Wolf, M. G. (2020). Thinking twice about sum scores. Behavior Research Methods, 1-19. https://doi.org/10.3758/s13428-020-01398-0 
Messick, S. (1994). The interplay of evidence and consequences in the validation of performance assessments. Educational Researcher, 23, 13-23. https://www.jstor.org/stable/1176219

Moore, S. A., Dowdy, E., Nylund-Gibson, K., \& Furlong, M. J. (2019). An empirical approach to complete mental health classification in adolescents. School Mental Health, 11, 438-453. https://doi.org/10.1007/s12310-019-09311-7

Moore, S. A., Mayworm, A. M., Stein, R., Sharkey, J. D., \& Dowdy, E. (2019). Languishing students: Linking complete mental health screening in schools to Tier 2 intervention. Journal of Applied School Psychology, 35, 257-289. https://doi.org/10.1080/15377903.2019.1577780

Moore, S. A., Widales-Benitez, O., Carnazzo, K. W., Kim, E. K., Moffa, K., \& Dowdy, E. (2015). Conducting universal complete mental health screening via student self-report. Contemporary School Psychology, 19, 253-267. https://doi.org/10.1007/s40688-015-0062-x

Muthén, B., Kaplan, D., \& Hollis, M. (1987). On structural equation modeling with data that are not missing completely at random. Psychometrika, 52, 431-462. https://doi.org/10.1007/BF02294365

R Core Team (2021). R: A language and environment for statistical computing. The R Foundation. https://www.r-project.org

Reise, S. P., Bonifay, W. E., \& Haviland, M. G. (2013). Scoring and modeling psychological measures in the presence of multidimensionality. Journal of Personality Assessment, 95, 129-140. https://doi.org/10.1080/00223891.2012.725437

Renshaw, T. L. (2015). A replication of the technical adequacy of the Student Subjective Wellbeing Questionnaire. Journal of Psychoeducational Assessment, 33, 757-768. http://doi.org/dpcq

Renshaw, T. L., \& Cohen, A. S. (2014). Life satisfaction as a distinguishing indicator of college student functioning: Further validation of the two-continua model of mental health. Social Indicators Research, 117, 319-334.https://doi.org/10.1007/s11205-013-0342-7 
Renshaw, T. L., Long, A. C. J., \& Cook, C. R. (2015). Assessing adolescents' positive psychological functioning at school: Development and validation of the Student Subjective Wellbeing Questionnaire. School Psychology Quarterly, 30, 534-552. https://doi.org/10.1037/spq0000088

Renshaw, T. L., \& Chenier, J. S. (2018). Further validation of the Student Subjective Wellbeing Questionnaire: Comparing first-order and second-order factor effects on actual school outcomes. Journal of Psychoeducational Assessment, 36, 392-397. http://doi.org/gdpmsc

Revelle, W. (2021) psych: Procedures for personality and psychological research. R package version 2.1.9. Northwestern University, Evanston, Illinois, USA. https://CRAN.Rproject.org/package=psych

Roberson, A. J., \& Renshaw, T. L. (2019). Initial development and validation of the Student Wellbeing Teacher-Report Scale. School Mental Health, 11, 578-599. https://doi.org/10.1007/s12310-018$\underline{09305-x}$

Rodriguez, A., Reise, S. P., \& Haviland, M. G. (2016a). Applying bifactor statistical indices in the evaluation of psychological measures. Journal of Personality Assessment, 98, 223-237. https://doi.org/10.1080/00223891.2015.1089249

Rodriguez, A., Reise, S. P., \& Haviland, M. G. (2016b). Evaluating bifactor models: Calculating and interpreting statistical indices. Psychological Methods, 21, 137-150.

\section{http://dx.doi.org/10.1037/met0000045}

Schmid, J., \& Leiman, J. M. (1957). The development of hierarchical factor solutions. Psychometrika, 22,

\section{3-61. https://doi.org/10.1007/BF02289209}

Seligman, M. E. P. (2018). PERMA and the building blocks of well-being. Journal of Positive Psychology, 13, 333-335. https://doi.org/10.1080/17439760.2018.1437466

Seligman, M. E. P., Ernst, R. M., Gillham, J., Reivich, K., \& Linkins, M. (2009). Positive education: Positive psychology and classroom interventions. Oxford Review of Education, 35, 293-311. https://doi.org/10.1080/030549809029 
Seligman, M. E. P., \& Adler, A. (2018). Positive education. Global happiness policy report, 52-73.

Seligman, M. E., \& Csikszentmihalyi, M. (2000). Positive psychology: an introduction. American Psychologist, 55(1), 5-14. https://doi.org/10.1037/0003-066X.55.1.5

Shankland, R., \& Rosset, E. (2017). Review of brief school-based positive psychological interventions: A taster for teachers and educators. Educational Psychology Review, 29, 363-392. https://doi.org/10.1007/s10648-016-9357-3

Steiner, M.D., \& Grieder, S.G. (2020). EFAtools: An R package with fast and flexible implementations of exploratory factor analysis tools. Journal of Open Source Software, 5(53), 2521. https://doi.org/10.21105/joss.02521

Suldo, S. M., \& Shaffer, E. J. (2008). Looking beyond psychopathology: The dual-factor model of mental health in youth. School Psychology Review, 37, 52-68. https://doi.org/10.1080/02796015.2008.12087908

Suldo, S. M., Thalji-Raitano, A., Kiefer, S. M., \& Ferron, J. M. (2016). Conceptualizing high school students' mental health through a dual-factor model. School Psychology Review, 45, 434-457. https://doi.org/10.17105/SPR45-4.434-457

Tian, L., Du, M., \& Huebner, E. S. (2015). The effect of gratitude on elementary school students' subjective well-being in schools: The mediating role of prosocial behavior. Social Indicators Research, 122, 887-904. https://doi.org/10.1007/s11205-014-0712-9

Tipton, E. (2014). Stratified sampling using cluster analysis: A sample selection strategy for improved generalizations from experiments. Evaluation Review, 37, 109-139.

\section{https://doi.org/10.1177/0193841X13516324}

Weissberg, R. P., Durlak, J. A., Domitrovich, C. E., \& Gullotta, T. P. (2015). Social and emotional learning: Past, present, and future. In J. A. Durlak, C. E. Domitrovich, R. P. Weissberg, \& T. P. Gullotta (Eds.), Handbook of social and emotional learning: Research and practice (p. 3-19). Guilford. 
Weist, M. D., Lever, N. A., Bradshaw, C. P., \& Owens, J. S. (Eds.). (2013). Handbook of school mental health: Research, training, practice, and policy. Springer.

Whitcomb, S. (2018). Behavioral, social, and emotional assessment of children and adolescents (4th ed.). Routledge.

Wickham, H., Averick, M., Bryan, J., Chang, W., McGowan, L. D. A., François, R., Grolemund, G., Hayes, A., Henry, L., Hester, J., Kuhn, M., Pedersen, T. L., Miller, E., Bache, S. M., Muller, K., Ooms, J., Robinson, D., Seidel, D. P., Spinu, V., ... Yutani, H. (2019). Welcome to the Tidyverse. Journal of Open Source Software, 4, 1686. https://doi.org/10.21105/joss.01686

Zhang, D. C., \& Renshaw, T. L. (2020). An examination of the relationship between personality and college student subjective wellbeing: A bifactor model approach. Journal of Happiness Studies, 21, 997-1014. https://doi.org/10.1007/s10902-019-00116-8 